\title{
Potenciales relacionados a eventos y funciones ejecutivas en niños y adolescentes
}

\section{Potentials related to events and executive functions in children and adolescents}

\author{
Bernarda Téllez Alanís \\ bernatellez@hotmail.com
}

Centro de Investigación Transdisciplinar en Psicología UAEM

\section{Resumen}

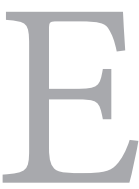

$\mathrm{n}$ la presente revisión teórica se examinan los trabajos iniciales de la técnica de los potenciales relacionados a eventos y se muestra su utilidad para el estudio de los procesos cognitivos durante el desarrollo. En un segundo momento, se esboza una breve historia del concepto de funciones ejecutivas y se enlistan algunos de los principales modelos que estudian dichas funciones en niños y adolescentes. Yen la tercera parte, se aborda el objetivo principal de este documento, el cual es mostrar que los potenciales relacionados a eventos proporcionan información útil en el estudio del desarrollo de algunas funciones ejecutivas, específicamente la inhibición, el cambio de la atención y el monitoreo del error.

Palabras clave: potenciales relacionados a eventos, inhibición, monitoreo, cambio atencional

\section{Summary}

he present theoretical review examines the initial work on the technique of event-related potentials and shows its usefulness for the study of cognitive processes during development. In a second moment, a brief history of the concept of executive functions is outlined and some of the main models that study these functions in children and adolescents are listed. In the third part, the main objective of this document is discussed, which is to show that event-related potentials provide useful information in the study of the development of some executive functions, specifically inhibition, change of attention and error monitoring.

Key words: event-related potentials, inhibition, monitoring, attentional change 


\section{Artículos de Revisión}

\section{Potenciales relacionados a eventos}

Se considera que los Potenciales Relacionados a Eventos (PREs) fueron descritos por primera vez por Pauline Davis en 1939. Esta investigadora reportó una onda asociada a la presentación de estímulos auditivos en adultos despiertos que fue directamente observada en el electroencefalograma (EEG). Posteriormente Walter, Cooper, Aldridge, McCallum y Winter (1964) describieron una onda negativa que se presentó entre un estímulo de aviso (click) y un estímulo de prueba (flash) ante el cual debía presionarse un botón. La onda fue denominada variación contingente negativa $\mathrm{u}$ onda de la expectativa (onda E). Para obtener este potencial se promediaron 12 segmentos del EEG. Al año siguiente Sutton, Braren, Zubin y John (1965) reportaron una onda positiva de mayor amplitud ante la incertidumbre de la modalidad sensorial de un estímulo prueba. Dichos autores presentaron pares de estímulos (aviso y prueba) en dos condiciones. En la primera, llamada condición segura, el estímulo prueba se presentaba en una sola modalidad sensorial (visual o auditiva) mientras que en la segunda, denominada condición insegura, el estímulo prueba se presentaba en una u otra modalidad sensorial en diferente porcentaje (77\% y $33 \%)$. Al comparar los potenciales de los estímulos prueba se encontró mayor amplitud en una onda positiva de amplitud máxima a los 300 milisegundos, en la condición insegura que en la condición segura, diferencia que fue más evidente cuando la probabilidad de presentación del estímulo fue del 33\%. Para la obtención de estos componentes se promediaron de 30 a 360 segmentos del EEG y se presentaron las ondas de 5 u 8 participantes.
Ahora bien, con respecto a la definición de los PREs se reconoce que éstos son cambios de voltaje (potencial) que ocurren en un momento antes, durante o después (relacionado) de un suceso en el mundo físico o de un proceso mental (evento) (Picton, 1988). El EEG se registra sobre el cuero cabelludo y tiene su origen en el reclutamiento sincronizado de potenciales postsinápticos excitatorios e inhibitorios de las dendritas apicales de las neuronas piramidales de la corteza cerebral. Algunos PREs se pueden observar directamente en el EEG (Davis, 1939), pero en su mayoría se obtienen con procesamiento de la señal electroencefalográfica a través del filtrado de frecuencias, la segmentación del EEG en torno al evento y el promedio de varios segmentos de acuerdo al tipo de estímulo o condición. Las características de los PREs son la polaridad, que puede ser positiva o negativa; la latencia que se refiere al tiempo en milisegundos desde el inicio de la presentación de un estímulo hasta cuando la onda alcanza su tamaño máximo y su amplitud que es la cantidad de energía de la onda (área bajo la curva en $\mathrm{mV}^{2}$ ). Por lo tanto los potenciales se nombran de acuerdo a su polaridad con una $\mathrm{P}$ (positiva) o una $\mathrm{N}$ (negativa) y con un número para señalar la secuencia de aparición $(1,2,3)$ o con la latencia en milisegundos (100, 200, 300, 600). También se pueden denominar de acuerdo al proceso cognitivo con el que se asocian (variación contingente negativa, potencial de la preparación motora, onda de la incongruencia semántica) o con una combinación de ambas nomenclaturas ( $\mathrm{P} 3$ de la novedad).

Varios PREs se han investigado en relación a distintos procesos cognitivos, por ejemplo: la N1 asociada a la atención selectiva tanto de modalidad auditiva como visual; la $\mathrm{P} 2$ relacionada 
al procesamiento sensorial temprano (auditivo); el componente $\mathrm{N} 2$ obtenido en la detección y respuesta a un estímulo blanco visual; la P3 que se asocia a procesos de actualización de la memoria o memoria de trabajo ya que tiene mayor amplitud ante los estímulos poco probables; la N4 relacionada a la incongruencia semántica; y el potencial de preparación motora que se refiere al aumento de un componente negativo previo a una respuesta motriz (Polich, 1993).

El empleo de los potenciales relacionados a eventos en el estudio de la cognición ha aumentado significativamente en los últimos 20 años, aún con la aparición de técnicas de imágenes cerebrales funcionales. Esto se debe en parte a las ventajas del EEG, las cuales son: a) una técnica no invasiva, es decir no se inyectan sustancias en el torrente sanguíneo ni se colocan electrodos en el interior de la cabeza; b) registra la actividad electromagnética cerebral asociada a los procesos postsinápticos de las neuronas, por tanto se obtiene información directa de la comunicación cerebral y no datos metabólicos como en otras técnicas; c) resolución temporal es en milisegundos, ésta es una de las ventajas más sobresalientes del EEG ya que en ese rango temporal suceden los procesos neuronales; $\mathrm{yd}$ ) el costo de los aparatos empleados es relativamente bajo por lo que los electroencefalógrafos están al alcance económico de varios laboratorios alrededor del mundo.

Sin embargo, también es necesario hablar de las desventajas, a saber: a) la resolución espacial es baja, es decir, no se pueden a realizar localizaciones precisas ni exactas del origen de la actividad cerebral como sí lo hacen otras técnicas; b) para obtener los PREs se requiere de un gran número de ensayos por lo que la sesión de registro puede ser larga y cansada para los participantes; y c) debido a que se realizan promedios de la actividad eléctrica, la precisión temporal se diluye afectando también la amplitud.

El tomar en cuenta tanto las ventajas y desventajas de la técnica nos permite ubicarnos claramente en el tipo de información que ésta provee y el tipo de interpretación que es permitida. Los PREs aportan información de tipo correlacional, es decir que tal conducta, proceso o tipo de estímulo se asocia con determinado potencial u onda y solamente eso. Los PREs no explican los procesos cognitivos sino que son un correlato electrofisiológico, y su presencia, ausencia o modificación contribuye, junto con otros indicadores conductuales y funcionales, a la elaboración de teorías explicativas complejas e integrales.

\section{Potenciales relacionados a eventos en el desarrollo}

El estudio de los PREs durante el desarrollo merece consideraciones especiales ya que en cada etapa de la vida existen diferentes condiciones anatómicas: el grosor y espesor de los huesos craneales (Lamm, Zelazo \& Lewis, 2006) y el cuero cabelludo cambian a lo largo de la vida así como también la densidad sináptica y la mielinización (Luna, Thulborn, Munoz, Merriam, Garver et al., 2001). Por otro lado se debe tomar en cuenta utilizar paradigmas sencillos que puedan ser aplicados a bebés, niños, adultos y hasta adultos mayores, es decir, que no requieran de respuestas verbales o motoras.

Uno de los procesos más estudiados en este campo es la percepción de rostros ya que al ser 
esta actividad tan fundamental en la vida social humana existe un interés por conocer desde qué edad el proceso se vuelve efectivo. Los PREs han aportado información al respecto, se reconoce un potencial negativo que aparece alrededor de los 170 milisegundos, denominado N170, asociado a la observación de caras en adultos (Bentin, Allison, Puce, Perez \& McCarthy, 1996). Ya en el área del desarrollo, Haan, Pascalis y Johnson (2002) presentaron rostros a niños de 6 meses (34 bebés) y a adultos entre 29 y 42 años (11 adultos) y confirmaron el hallazgo de la N170 en el gran promedio de los adultos, mientras que en los bebés esta onda se presentó pero con mayor amplitud y con mayor latencia, más allá de los 200 mseg. En la misma línea de trabajo, Taylor, Batty e Itier (2004) evaluaron varios grupos de niños desde los 4 a los 11 años, adolescentes de 12 a 15 años y adultos. En estos últimos se replicó la presencia de la N170 ante los rostros mientras que en los niños esta onda se presentó pero con un gran retraso en la latencia, en algunos grupos después de los $250 \mathrm{mseg}$. En su conjunto estos hallazgos señalan que el indicador electrofisiológico de la percepción de rostros se encuentra desde muy temprana edad (6 meses) lo cual se relacionada con la capacidad de los niños de discriminar caras, sin embargo la madurez del proceso puede requerir un poco más de tiempo. Además el aumento de la amplitud de la N170 fue interpretado como un mayor reclutamiento de regiones posinápticas o cómo un proceso originado por la mayor densidad neuronal de los pequeños.

Por otro lado, durante el desarrollo también se han estudiado los componentes asociados a la percepción de sonidos. En adultos se ha detectado la presencia de un grupo de ondas $\mathrm{P} 1$,
$\mathrm{N} 1$ y P2, mientras que en los niños pequeños sólo se presenta la P1 y la N2. Entre un año y cuatro años de edad la $\mathrm{P} 1$ es el componente más robusto, de los 3 a los 6 años la N2 se vuelve el más prominente lo que continúa hasta la adolescencia, por último la N1 emerge hasta los 4 años (Ceponiene, Rinne \& Naatanen, 2002). En la misma línea de investigación Kushnerenko, Ceponiene, Balan, Fellman, Huotilainen y Naatanen (2002) reportaron la presencia de una onda negativa asociada a la incongruencia (MMN Mismatch Negativity) en bebés recién nacidos. Dichos autores realizaron un estudio longitudinal y midieron a 12 bebés cada tres meses hasta que alcanzaron el año de vida y asociaron esta onda a la capacidad de distinguir sonidos.

La evidencia presentada nos indica que los PREs pueden ser utilizados exitosamente en el estudio de los procesos perceptuales y cognitivos durante el desarrollo, debido principalmente a dos de sus ventajas: que no es una técnica invasiva y que no se requiere de una respuesta activa (Ceponiene et al., 2002). A continuación se revisará el tema de las funciones ejecutivas y posteriormente se abordará el propósito central de este documento que es describir y discutir varios estudios con PREs que han evaluado algunas funciones ejecutivas.

\section{Funciones ejecutivas}

\section{Reseña del estudio de los efectos de las lesiones frontales}

A finales del siglo XIX el médico inglés David Ferrier presentó una revisión de casos clínicos de lesión prefrontal descritos por varios médicos europeos: en todos los pacientes 


\section{Artículos de Revisión}

reportados se observó una ausencia de efectos motores o sensoriales, pero en algunos casos los médicos detectaron algunas alteraciones psicológicas como letargo, falta de curiosidad o una especie de demencia (Ferrier, 1878; Téllez, 2013). Más adelante Moritz Jastrowitz en 1888 y Hermann Oppenheim en 1890 indicaron que los pacientes con tumores en el polo frontal derecho presentaron un tipo de demencia caracterizada por estados de euforia (Luria, 1980), aunque también mencionaron que otros pacientes más bien parecían deprimidos (Finger, 2001).

Ya en el siglo XX, Ernest Feuchtwanger, médico alemán, publicó en 1923 la comparación de observaciones conductuales realizadas en 200 soldados heridos con bala en la región frontal con los datos obtenidos en 200 soldados heridos en otra región de la cabeza. Los cambios que aparecían tras lesión frontal fueron modificaciones en la atención, la motivación, la iniciativa y en las reacciones emocionales (Teuber, 2009). En 1927, otro médico alemán que emigró a los Estados Unidos, Kurt Goldstein, publicó que los pacientes con lesiones frontales tenían problemas para desarrollar la actitud abstracta (razonamiento abstracto, resistencia a la sugestión, iniciativa, previsión, autoconsciencia, flexibilidad conductual y cognitiva, y la habilidad para analizar una situación compleja), mientras que conservaban la actitud concreta (Tranel, Anderson \& Benson, 1994). Para algunos autores el término de actitud abstracta es un antecedente del término de funciones ejecutivas.

Por otro lado, el célebre médico ruso, Alexander Romanovich Luria, observó a cientos de soldados de la segunda guerra mundial con lesiones en diferentes regiones cerebrales, incluyendo a aquellos que presentaban lesiones frontales. Además él propone que las funciones psicológicas tienen como sustrato fisiológico a la acción coordinada del SNC al cual divide en tres bloques. El primer bloque es el encargado del tono cortical, el segundo de la recepción, análisis, síntesis y almacenamiento de la información que llega del exterior y el tercer bloque, denominado ejecutivo, es el encargado de la programación ejecución y control de la acción (Luria, 1988).

\section{Modelos de funciones ejecutivas}

Muchos fueron los médicos que aportaron evidencias sobre las alteraciones en el comportamiento y en la personalidad que aparecían tras una lesión prefrontal. Es así como fueron apareciendo conceptos como "actitud abstracta" y "bloque ejecutivo" que describían las funciones superiores asociadas a las funciones frontales. Posteriormente, en 1980, la psicóloga norteamericana Muriel Lezak, retoma el concepto del bloque "ejecutivo" en un documento presentado en un congreso en Australia, el trabajo fue sobre la iniciación, la planeación y las capacidades ejecutivas. Dos años después Lezak (1982) publica un trabajo sobre la dificultad para valorar las funciones ejecutivas, a las cuales define como "las capacidades requeridas para formular metas, la planeación y la ejecución efectiva de los planes". Además indicó que dichas funciones ejecutivas son esenciales para la conducta independiente, creativa y socialmente útil, que se alteran tras una lesión frontal y señaló que en esos momentos no existían pruebas para evaluarlas clínicamente, lo cual es difícil per se debido a la naturaleza de las mismas.

Adicional a este modelo surgieron otros modelos para explicar el funcionamiento 


\section{Artículos de Revisión}

ejecutivo: El modelo del ejecutivo central de Baddeley y Hitch (1974), el modelo del sistema atencional supervisor de Norman y Shallice (1986), el modelo jerárquico de la actividad cerebral de Stuss y Benson (1986), pero todos ellos desarrollados a partir del análisis de casos de pacientes adultos.

Posteriormente, en la década de los 90's del siglo XX, comienzan a surgir modelos creados específicamente desde la perspectiva del desarrollo. Para Welsh, Pennington y Groisser (1991) la función ejecutiva es la conducta dirigida a una meta, incluyendo la planeación, el empleo flexible de estrategias, la búsqueda organizada y el control de impulsos. Ellos evaluaron a 100 estudiantes entre 3 y 12 años y encontraron que a) el nivel de ejecución de los adultos fue alcanzado a diferentes edades, dependiendo de la tarea, a los 6 años, a los 10 y en la adolescencia; b) que las medidas obtenidas en las pruebas aplicadas se agruparon en tres factores: velocidad de respuesta, mantenimiento del set y planeación; y c) que la mayoría de las medidas de funciones ejecutivas no correlacionaron con la inteligencia.

Desde una perspectiva más aplicada, Gioia, Isquith, Roth, Guy, Kenworthy y Espy (2000) diseñaron un inventario, que deben contestar padres o maestros, para detectar problemas en conductas asociadas a las funciones ejecutivas en niños y adolescentes (de 5 años hasta 18 años). Estos autores distinguen ocho funciones ejecutivas agrupadas en dos índices. El primer índice es denominado regulación conductual y el segundo es el de metacognición. El índice de regulación conductual se refiere al funcionamiento en la actividad concreta, de las acciones y relaciones con los objetos, posee tres componentes: la inhibición que es la capacidad para detener respuestas automáticas, preponderantes o previamente activadas; la flexibilidad que es la capacidad para alternar eficientemente el foco de atención; y el control emocional que se refiere a la modulación adecuada de las respuestas emocionales. Por otro lado el índice de metacognición hace referencia al conocimiento del propio funcionamiento cognitivo o mental y está compuesto por las escalas de monitoreo, organización de materiales, planeación, memoria de trabajo e iniciación. El monitoreo se refiere a la capacidad de revisar la actividad realizada, a evaluar el desempeño propio; la organización de materiales se refiere a la capacidad de ordenamiento de objetos concretos en entornos específicos; la planeación es la capacidad de organizar una serie de pasos para resolver una tarea; la memoria de trabajo garantiza el procesamiento de información adecuado, es decir el manejo de la información útil y la eliminación de la información no útil; mientras que la iniciación se refiere a la capacidad para comenzar una actividad voluntaria y a la generación de ideas.

Ahora bien, en el trabajo con niños pequeños también han surgido posturas teóricas de lo que son las FE. Anderson (2001) define a las funciones ejecutivas como las habilidades necesarias para la actividad propositiva, dirigida a una meta, además la autora indica que estas funciones están mediadas por la corteza prefrontal y señala que aunque en los niños pequeños aún no está desarrollada dicha corteza, en ellos ya se pueden encontrar tales funciones. Anderson también establece que en la evaluación neuropsicológica de las FE debe incluir la planeación, la solución de 


\section{Artículos de Revisión}

problemas, el pensamiento abstracto, formación de conceptos y la flexibilidad mental.

Otro grupo influyente en el estudio del desarrollo de las FE es dirigido por David Zelazo, quien indica que las FE son funciones separadas: la planeación, la toma de decisiones, la inhibición de impulsos y la autorregulación (Zelazo \& Muller, 2002). Más adelante también incluyen otros componentes como el mantenimiento de la información en la memoria de trabajo, la inhibición de respuestas dominantes, el cambio apropiado de la atención y la conservación de la misma con el propósito de asegurar la acción dirigida hacia un fin (Blair, Zelazo y Greenberg, 2005).

Para Dawson y Guare (2004), quienes desarrollaron un programa de atención de las funciones ejecutivas para niños y adolescentes, las habilidades del pensamiento requeridas para alcanzar las metas son la planeación, la organización, el manejo del tiempo, la memoria de trabajo y la metacognición, adicionalmente se requiere de la inhibición de respuestas, la autorregulación del afecto, la iniciación de la tarea, la flexibilidad y las persistencia dirigida a la meta.

Ya desde una perspectiva más integral, para aplicar estos modelos en el contexto educativo, Diamond y Lee (2011) proponen que los niños requieren creatividad, flexibilidad, autocontrol y disciplina para ser exitosos, que esas son las funciones ejecutivas y que dependen de un circuito en el que la corteza prefrontal tiene un papel central. Estas autoras distinguen dos tipos de funciones: las nucleares que incluyen la flexibilidad cognitiva, la inhibición (autocontrol, autorregulación) y la memoria de trabajo; y las funciones ejecutivas más complejas que son resolución de problemas, el razonamiento y la planeación. Resaltan la importancia de las FE ya que son más influyentes en el rendimiento académico que el coeficiente intelectual, así como también son determinantes en otros aspectos de la vida cómo la elección de carrera, el matrimonio y la salud mental y física.

Asimismo se han desarrollado pruebas específicas para el trabajo con niños pequeños. Por ejemplo, para evaluar la flexibilidad cognitiva se usan las tareas tipo Wisconsin Card Sorting Test, en las que se trabaja sólo con el criterio de la forma y el color y además se le da la regla específica al niño, quien no la tiene que detectar como en la versión de adultos, y también hay versiones a color o de otro tipo del Trail Making test. Para la inhibición se emplean tareas como día-noche; el osito de peluche y el dragón; y la de los susurros; en las tres anteriores se le pide al niño que detenga una respuesta dominante pero empleando estímulos sencillos y comunes para ellos.

En el idioma inglés algunas de la pruebas para evaluar las funciones ejecutivas en niños son: la batería NEPSY que evalúa la planeación, la flexibilidad cognitiva, la impulsividad, la vigilancia, la atención auditiva selectiva, el monitoreo, la autorregulación y la resolución de problemas (Korkman, Kirk \& Kemp, 1998) y la Behavioural assessment of the disexecutive syndrome for children (BADS-C) (Emslie, Wilson, Burden, Nimmo-Smith \& Wilson, 2003).

En países de habla castellana contamos con la Batería Neuropsicológica de las Funciones Ejecutivas y Lóbulos Frontales (BANFE) que 


\section{Artículos de Revisión}

es para niños y adultos, de 5 a 80 años (Flores, Ostrosky y Lozano, 2012), la Evaluación neuropsicológica de las funciones ejecutivas en niños (ENFEN) (Portellano, MartínezArias \& Zumárraga, 2012) que es para niños de 6 a 12 años, y la subescala Torre México de la Evaluación Neuropsicológica Infantil (Matute, Roselli \& Ardila, 2010).

\section{PREs y funciones ejecutivas en niños y adolescentes}

En adultos, las funciones ejecutivas que principalmente se han evaluado mediante los PREs son: la memoria de trabajo (Ruchkin, Johnson, Grafman, Canoune, \& Ritter (1992), la inhibición (Garavan, Ross, Murphy, Roche, \& Stein, 2002; Jonkman, Lansbergen \& Stauder, 2003), el monitoreo del conflicto (Garavan et al., 2002; Nieuwenhuis, Yeung, Van Den Wildenberg, \& Ridderinkhof, 2003) y el cambio atencional (Kieffaber \& Hetrick, 2005). En los niños sucede algo similar, se ha estudiado la inhibición, el monitoreo del conflicto y el cambio atencional. Cabe mencionar que ni en adultos ni en niños o adolescentes se han evaluado procesos ejecutivos como la motivación o la planeación ya que por los requerimientos de la técnica no se han implementado paradigmas adecuados para registrar los PREs.

Un componente de los PREs muy estudiado en el tema de la inhibición es el N2 y existe un debate si éste refleja la inhibición de respuestas (Jonkman et al., 2003) o el monitoreo del conflicto (Nieuwenhuis et al., 2003). Sin embargo, de manera general se acepta que está asociado a procesos del control cognitivo (Lamm, Zelazo y Lewis, 2006). En adultos se han reportado generadores de esta onda en el cíngulo (Nieuwenhuis et al., 2003) y en la corteza orbitofrontal derecha (Bokura, Yamaguchi, \& Kobayashi, 2001).

En niños, Lamm et al. (2006) evaluaron el control cognitivo requerido para inhibir una respuesta motora mediante el análisis del componente N2. En su estudio registraron la actividad eléctrica de 33 participantes de 7 a 16 años mientras los participantes realizaban una tarea tipo go-no go que consistía en responder ante letras que se presentaban de manera secuencial en una pantalla, sin embargo se debía inhibir su respuesta cuando se presentaba una letra repetida. Adicionalmente los niños realizaron otras tareas asociadas al funcionamiento cognitivo: la Iowa Gambling Task (IGT), la tarea Stroop, una tarea de descuento retrasado y los dígitos inversos. Los PREs fueron obtenidos ante los estímulos go (responder) y ante los no go (no responder) y se compararon por edad (niños y adolescentes) pero también de acuerdo a la ejecución de los participantes en las tareas cognitivas (buena y mala ejecución). De manera general, los niños presentaron un retraso en la latencia del componente $\mathrm{N} 2$ independientemente de su ejecución. Mientras que la mala ejecución en dichas tareas se asoció a una mayor amplitud en dicho componente sin importar la edad. Además también fueron calculados los generadores del componente $\mathrm{N} 2$ los cuales se encontraron en las regiones orbitofrontales y en el cíngulo. En específico, en los dos grupos con buenas ejecuciones en la IGT y en la tarea Stroop se encontró un generador en el cíngulo anterior. Además, se encontró un generador en la región orbitofrontal izquierda en los niños que tenían malas ejecuciones mientras que en el grupo de adolescentes con buenas ejecuciones se 


\section{Artículos de Revisión}

encontró en la región orbitofrontal derecha. Los autores interpretaron estos hallazgos señalando que la disminución de la amplitud de la N2 muestra el desarrollo del control cognitivo, no así la disminución de la latencia que estaría más asociada al desarrollo físico. Funcionalmente la disminución de la amplitud de la N2 estaría mostrando un cambio de las regiones subyacentes a dicho componente. Sin embargo, estos hallazgos deben replicarse con una muestra más homogénea entre los grupos ya que en este estudio la cantidad de participantes por grupo estuvo desequilibrada (niños pobre ejecución 13, niños buena ejecución 5, jóvenes pobre ejecución 9 y adolescentes buena ejecución 6) y sólo así se consolidará la noción de que la disminución de amplitud de la N2 es un indicador del desarrollo del control cognitivo.

Por otro lado, tradicionalmente la inhibición de respuestas se ha evaluado con tareas en las que hay que aprender el estímulo específico ante el cual no dar una respuesta. Para tratar de evaluar la inhibición en una tarea que no dependiera de la detección de un estímulo específico sino de la resolución de otra tarea cognitiva, Barona (2011) realizó un estudio en el que presentó pares de un símbolo y una palabra, con dos condiciones: congruente e incongruente. Los participantes debían inhibir su respuesta ante el par congruente (J-FELIZ) o ante el incongruente (L-FELIZ) y también se registraron los PREs. Los resultados mostraron que la tarea fue fácil ya que las ejecuciones estuvieron por arriba del $90 \%$ tanto en el grupo de niños (11 años) como en el de adolescentes (15 años), sin embargo fue más difícil inhibir la respuesta ante los pares congruentes lo cual es similar a lo reportado por Banfield, Lugt y Munte (2006). Respecto a los PREs, en el grupo de adolescentes si se presentó la N2 con mayor amplitud ante los ensayos en los que se inhibieron correctamente la respuestas, pero en el grupo de niños no se presentó diferencia en la N2 entre los ensayos en que hubo respuesta y en los que no. Además esto no varió de acuerdo a la condición congruente o incongruente. La ausencia de la diferencia de la $\mathrm{N} 2$ en el grupo de niños difiere de lo reportado en estudios previos (Jonkman et al., 2003; Lamm et al., 2006), pero coincide con los resultados del estudio de Davis, Bruce, Snyder y Nelson (2003) quienes no encontraron este componente ni en los niños ni en los adultos que evaluaron. Dichos autores declararon que esta ausencia de la N2 podía deberse a una mayor propagación de la P3 que obtuvieron ya que ésta fue muy robusta. En el estudio de Barona (2011) se obtuvo una onda P2, previa a la N2, muy amplia en los niños en comparación con los adolescentes, sin embargo, no se consideró que eso haya originado la ausencia de diferencia de la N2 ya que ésta si se presentó (Fig. 1) sólo que no hubo diferencia entre responder y no responder. La autora comentó que ese resultado podía deberse a que los niños involucraron más recursos cerebrales para resolver la tarea y así obtener la misma eficiencia que los adolescentes, esto sería similar a lo que lo propone la hipótesis de mayor extensión de la activación funcional en adultos mayores que en jóvenes, aun cuando ambos grupos presenten la misma ejecución (Cabeza, 2002). 
Niños
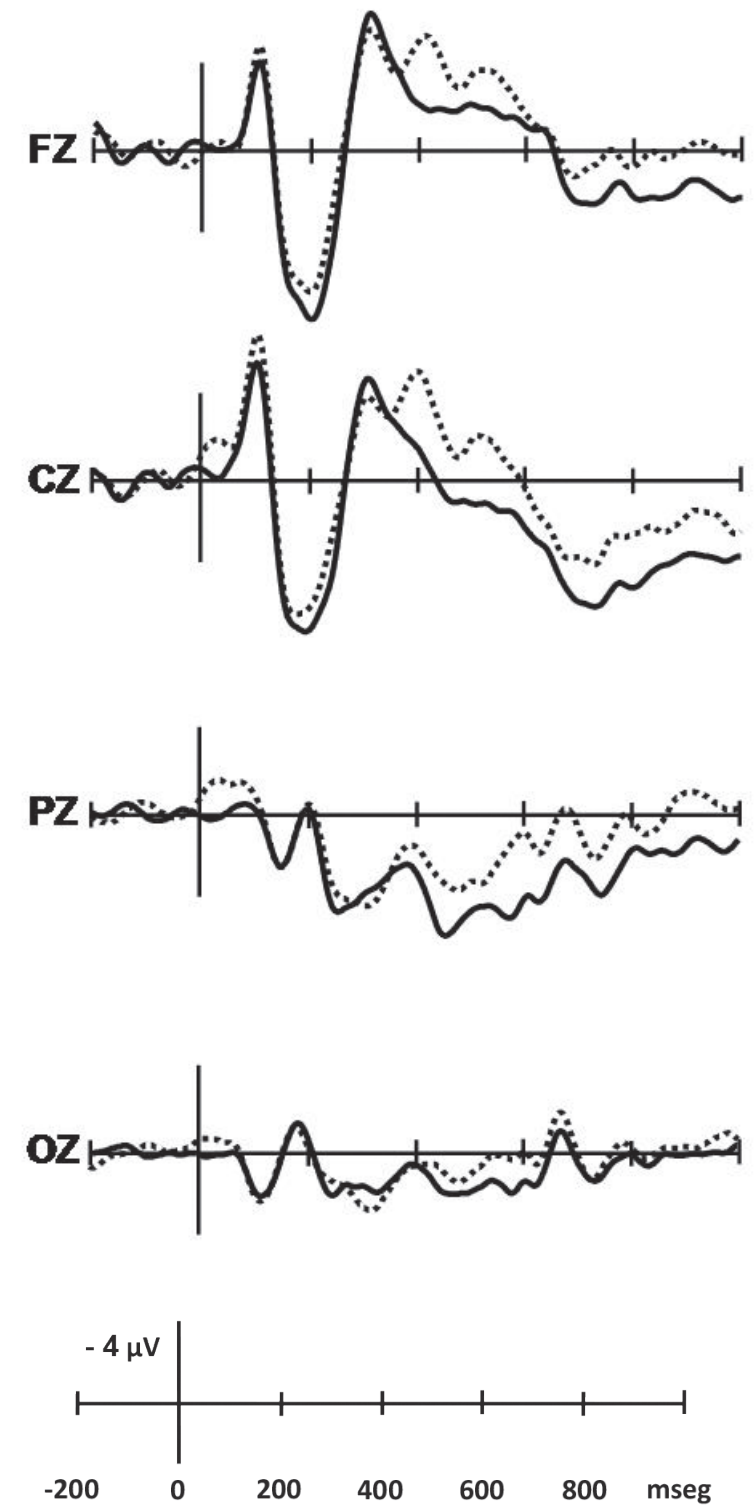

Adolescentes

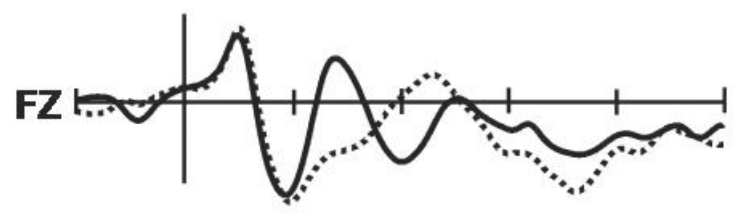

CZ

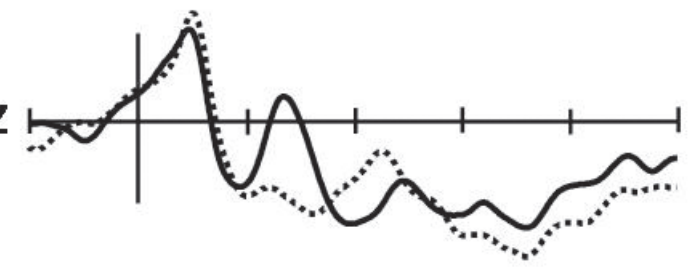

$\mathbf{P Z}$
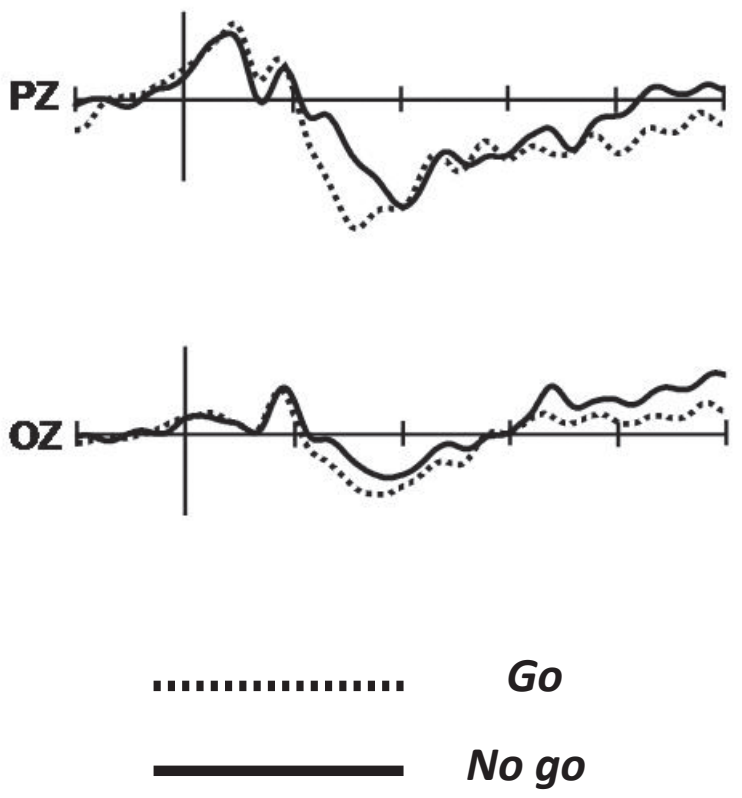

Figura 1. PREs de niños y adolescentes en una tarea de inhibición de respuestas desencadenada por la detección de una relación semántica. Cortesía de Barona (2011). 


\section{Artículos de Revisión}

Adicionalmente, en dicho estudio se debe considerar que los procesos de detección de una incongruencia semántica se evidencian con el componente N400. En términos temporales, en esa tarea no se podía esperar que primero se activará la inhibición (200 mseg) cuando aún no se presenta el componente asociado a la evaluación semántica (400 mseg). Por tanto, en este estudio los procesos de inhibición esperados deberían ser posteriores, evidenciados entre los 301-400 mseg, y efectivamente en dicha temporalidad sí hubo diferencias entre los ensayos go y los no go tanto en niños como en adolescentes. Así, el resultado del estudio citado contribuye con la noción de que en tareas de cognición la N2 podría estar reflejando procesos de mayor control atencional para desencadenar correctamente una inhibición conductual más que procesos inhibitorios per se, los cuales podrían estar asociados más tardíamente a la P3.

Con respecto al estudio de otros procesos ejecutivos, encontramos que uno de los paradigmas más utilizados para medir la flexibilidad cognitiva son las tareas tipo switch (Cepeda et al., 2001). Manzi, Nessler, Czernochwoski y Friedman (2011) estudiaron los procesos del control cognitivo anticipatorio con una tarea tipo switch en 14 niños (9-10 años), 14 adolescentes (13-14 años) y 20 jóvenes adultos (20-27 años). Los autores presentaron estímulos $(1,3,111,333)$ en la pantalla y la tarea fue resolver bloques puros donde la pregunta era "que número" se presentaba o "cuantas veces está el número", las respuestas siempre eran 1 o 3. Posteriormente presentaron bloques mixtos en los que aparecían combinadas las tareas, en el bloque mixto a un ensayo podía seguirle otro con la misma tarea (sin cambio) u con la otra tarea (con cambio). Los autores evaluaron los PRE asociados a la combinación de tareas comparando los ensayos de los bloques puros con los ensayos sin cambio de los bloques mixtos, esto con el objeto de evaluar la actualización de la configuración de la tarea (task-set updating). Encontraron una actividad positiva mayor en los ensayos sin cambio en comparación a los ensayos puros, además esto presentó diferencias de acuerdo a la edad: en los adultos y en los adolescentes el efecto comenzó más tempranamente y duró más (200-600 mseg) que en los niños (300-600 mseg). Además este efecto en los adultos fue constante y se presentó en derivaciones frontales, mientras que en los adolescentes no fue continuo y en los niños el efecto fue más corto (300-500 mseg). Los autores mencionan que es ampliamente reconocido que se requiere de la participación de la corteza prefrontal para garantizar un control ejecutivo eficiente por lo que esta ausencia del efecto de los electrodos frontales puede asociarse a la menor eficiencia en la ejecución presentada por los niños en los bloques mixtos en comparación con los puros y aun cuando reconocen que los PREs no tiene gran resolución espacial, ellos indican que es válido hacer la especulación ya que es compatible con la evidencia de que la corteza frontal va madurando conforme el desarrollo (Diamond, 2002) y también es compatible con estudios que demuestran que hay activación frontal reducida en tareas tipo switch en niños y adolescentes empleando resonancia magnética funcional (Crone, Donohue, Honomichl, Wendelken \& Bunge, 2006).

Manzi et al. (2011) también compararon los PREs de los ensayos sin cambio con los ensayos con cambio de los bloques mixtos para comparar el 


\section{Artículos de Revisión}

efecto de la reconfiguración del set de la tarea (taskset reconfiguration). Ellos reportaron una actividad positiva ( 300) mayor ante los ensayos con cambio en comparación a los ensayos sin cambio, pero no hubo diferencias en este efecto de acuerdo a la edad. Otros estudios ya habían reportado la ausencia de diferencias en este efecto durante el desarrollo y esto se había interpretado como una evidencia de que los procesos de control cognitivo necesarios para coordinar y reconfigurar una tarea son igual de eficientes en los grupos examinados (Crone, Ridderinkhof, Worm, Somsen \& van der Molen, 2004) o son más estables (Reimers \& Maylor, 2005). Lo que sí obtuvieron en este estudio fue una diferencia en el inicio del efecto, en los niños fue hasta los $400 \mathrm{mseg}$, mientras que en los adolescentes y adultos fue a los $300 \mathrm{mseg}$, falta aclarar si esta diferencia refleja los cambios físicos o si es una evidencia del desarrollo del proceso de reconfiguración. Además es importante precisar que ondas positivas con similar topografía y latencia se han asociado a los procesos involucrados en la reconfiguración de la tarea (Tieges, Snel, Kok, Plat \& Ridderinkhof, 2007).

Este importante estudio de Manzi et al. (2011) subraya la importancia del componente positivo que alcanza las derivaciones frontales en los adultos cuando se compara una tarea aislada con una tarea mezclada. Para los autores este componente sería un correlato de la memoria de trabajo y mencionan que se le ha otorgado menos atención que al componente positivo centroparietal (efecto de los costos por switch), el cual estaría más asociado a procesos de reconfiguración de la tarea.

Por otro lado, Davies, Segalowitz y Gavin (2004) realizaron un estudio para conocer el desarrollo de los componentes asociados al monitoreo de los errores, registrando la onda negativa relacionada al error (Error-related negativity ERN) y la onda positiva asociada al error $\left(\mathrm{P}_{\mathrm{e}}\right)$. Ellos registraron el EGG de participantes desde los 7 hasta los 18 años así como también a un grupo de adultos jóvenes mientras realizaban la tarea delos flancos deEriksen que consiste en proporcionar una respuesta ante un estímulo central en una condición congruente (HHHHH) y ante una incongruente (SSHSS). La primera condición es sencilla, pero en la segunda se debe inhibir la interferencia del contexto, siendo esta última la que requiere de un control ejecutivo. Los autores encontraron que el número de errores disminuía conforme aumentaba la edad y que la ERN casi nunca se presentaba en los niños pequeños (7-12), algunas veces se presentaba en los adolescentes (13-17 años) mientras que en los adultos siempre estaba presente. En cambio, la $\mathrm{P}_{\mathrm{e}}$ siempre estuvo presente a lo largo del desarrollo. Estos datos apoyan la idea de que la ERN tiene su generador en el cíngulo anterior ( $\mathrm{O}^{\prime} \mathrm{Connell}$, Dockree, Bellgrove, Kelly, Hester, Garavan et a., 2007) y que éste se continúa desarrollando a lo largo de la adolescencia. Por otro lado, los autores reportaron que los niños se daban cuenta de que cometían errores por lo que mencionan que aún es necesario esclarecer el papel de los PRE en el monitoreo del error, ya que la $\mathrm{P}_{\mathrm{e}}$ sí se presenta desde la infancia.

Ahora bien, Hillman, Buck, Themanson, Pontifex y Castelli (2009) investigaron si la salud física, en términos de capacidad aeróbica, está asociada a un mejor desempeño en una tarea de control cognitivo registrando precisamente la ERN y la $\mathrm{P}_{\mathrm{e}}$. Estos autores también utilizaron la tarea de los flancos de Eriksen. En los adultos ya se ha encontrado una relación pequeña pero 


\section{Artículos de Revisión}

significativa entre el ejercicio y la ejecución cognitiva (Etnier, Salazar, Landers, Petruzzello, Han \& Nowell, 1997). En el estudio de Hillman et al. participaron 38 niños con una media de edad de 9.4 años: 19 con capacidad aeróbica alta y 19 con capacidad aeróbica baja. Los resultados mostraron que el grupo con mayor capacidad realizó un mayor número de respuestas acertadas (82\%) que el grupo con menor capacidad aeróbica (75\%). Además ellos obtuvieron los PREs de las respuestas correctas a partir de la presentación del estímulo: la N2 (240-425 mseg), la P3 (375-675 mseg) y una onda lenta (675-975 mseg). También compararon los potenciales asociados a los errores de comisión: la ERN (0200 mseg post-respuesta) y $\mathrm{P}_{\mathrm{e}}$ (200-500 mseg post-respuesta). Sus resultados mostraron que la N2 presentó mayor amplitud ante los ensayos incongruentes que ante los congruentes, pero no hubo diferencia alguna de acuerdo al nivel de capacidad aeróbica; la P3 fue mayor en el grupo de mayor capacidad aeróbica, lo cual fue interpretado por los autores de acuerdo a las teorías contemporáneas que señalan que una mayor amplitud de la P3 se asocia a un incremento en la distribución de los recursos atencionales durante la codificación del estímulo (Polich \& Kok, 1995). La onda lenta no presentó diferencias entre los grupos. La ERN obtenida de los errores de comisión fue de menor amplitud en el grupo de mayor capacidad aeróbica que ante el grupo de menor capacidad aeróbica mientras que en la $\mathrm{P}_{\mathrm{e}}$ ocurrió exactamente lo contrario, mayor amplitud positiva en el grupo con mejor capacidad aeróbica; además la amplitud de esta onda correlacionó con una mejor ejecución conductual después de un error. Ante estos resultados los autores sugieren que la salud física puede estar relacionada con una mayor efectividad en el sistema de monitoreo de la acción ya que una reducción de la amplitud de la ERN puede reflejar una reducción en el umbral de evaluación para aumentar el control top-down, promoviendo un incremento en la integridad de la distribución de la atención; además que el aumento de la amplitud del componente $\mathrm{P}_{\mathrm{e}}$ se asociaría una mejor evaluación del error y a una mayor regulación del proceso de control cognitivo en los ensayos subsecuentes. Así este estudio demuestra el efecto positivo que induce la salud física, específicamente en términos de capacidad aeróbica, en los procesos cognitivos como la codificación del estímulo reflejado por la $\mathrm{P} 3$, en un aspecto ejecutivo que es el monitoreo del conflicto asociado a los componentes ERN y $\mathrm{P}_{\mathrm{e}}$, así como también en el aumento de la precisión de la ejecución durante el desarrollo.

La utilización de los PREs para investigación de funciones cognitivas superiores también se puede realizar con fines educativos. Por ejemplo, Stevens, Lauinder y Neville (2009), interesados en los estudios que indican que existen deficiencias conductuales en aspectos de la atención y de la función cognitiva en función del estatus socieconómico (ESE) familiar (Farah et al., 2006), registraron los PREs en una tarea de atención selectiva auditiva en niños cuyas madres tenían bajos niveles de educación (sin ninguna experiencia de educación superior) y niveles altos (alguna experiencia en la educación superior), tomando el nivel educativo de las madres como un indicador del ESE. Dicho estudio tuvo el objetivo de analizar si existían diferencias en la atención selectiva y sostenida en modalidad auditiva en las etapas tempranas del procesamiento ( $\sim 00 \mathrm{mseg})$. Participaron 32 
niños entre 3 y 8 años de edad, la tarea consistió en presentar 2 historias diferentes al mismo tiempo, una en la bocina derecha y otra en la izquierda. Los niños debían atender sólo a una de las dos historias, al mismo tiempo se presentaban imágenes de la historia en una pantalla $y$, además, se presentaban de manera auditiva los estímulos prueba, el lingüístico (sílaba $b a$ ) y el no lingüístico (zumbido), en una de las dos bocinas manera aleatoria cada 200, 500 o 1000 mseg. Al final de la tarea se les hacían 3 preguntas básicas de comprensión a los niños. Ellos obtuvieron los PREs de los estímulos prueba (sílaba y zumbido) en las dos condiciones (atendido y no atendido). En estudios similares con adultos se presenta una onda positiva temprana $(\mathrm{P} 1)$ seguida por una onda negativa alrededor de los $100 \mathrm{mseg}$ (N1), en cambio, en los niños se presenta una onda positiva amplia (100-300 mseg) (Sanders, Stevens, Coch \& Neville, 2006). En este estudio se presentó dicha onda positiva amplia, la cual no presentó diferencias entre el estímulo atendido y el no atendido solamente en el grupo de niños con madres de nivel escolar bajo. Lo anterior fue interpretado como una evidencia de que en esos niños hay una falla para filtrar información irrelevante, lo cual incide directamente en su vida escolar y cotidiana. Dicho estudio aporta información relevante para la detección de problemas en la atención con el objetivo de iniciar programas de intervención temprana.

Ahora bien, para evaluar la focalización voluntaria bajo condiciones de carga de la memoria de trabajo en niños de 9 y 10 años comparados con adultos, Ruhnau, Wetzel, Widmann y Schroger (2010) aplicaron un paradigma de distracción auditivo-visual que consiste en la presentación simultánea de sonidos estándares y de sonidos ambientales, seguidos por un estímulo visual (target). Además la tarea contaba con dos condiciones, los participantes evaluaron la posición del estímulo visual (0back, carga baja) o comparaban la posición del estímulo actual con la de dos ensayos anteriores (2-back, carga alta). En este estudio analizaron la MMN, la P3a, la negatividad de la reorientación (Reorienting negativity $\mathrm{RON}$ ) y la P3b visual. Sus resultados mostraron que en ambas cargas de memoria los sonidos novedosos disminuyeron la ejecución en los adultos mientras esos mismos sonidos mejoraron la ejecución en los niños. En la temporalidad de la MMN (90-250 mseg) los niños mostraron una onda positiva ante los sonidos novedosos mientras que en los adultos hubo una ausencia de la MMN. El cambio de la atención hacia la tarea novedosa irrelevante, reflejada por la P3a, fue comparable entre ambos grupos. Además los adultos mostraron una reubicación más eficiente de la atención (reflejada por la $\mathrm{RON}$ ) que los niños en la condición de carga alta. La P3b asociada al estímulo visual mostró menor amplitud en ambos grupos, cuando el sonido precedido fue novedoso. Los hallazgos de esta investigación aportan al estudio del desarrollo del procesamiento de los estímulos novedosos: a) que éstos pueden producir efectos contrarios en niños y en adultos sobre una tarea primaria visual; b) la presencia de una onda positiva ante los estímulos novedosos en niños más que una MMN; y c) revela los efectos de estímulos auditivos novedosos sobre el procesamiento visual.

\section{Conclusión}

El objetivo del presente documento fue abordar el estudio de las funciones ejecutivas mediante el análisis de los PREs en razón de que esta es 
una técnica que tiene varias ventajas a su favor y que ha demostrado ser útil en la investigación de diversos procesos cognoscitivos durante el desarrollo, por ejemplo en la percepción de rostros y en la percepción de sonidos. Por tanto esta técnica ha sido utilizada para el estudio de las funciones cognitivas supramodales como lo son las FE. A pesar de la existencia de cambios físicos y funcionales durante el desarrollo, la información de los componentes obtenidos en manipulaciones experimentales específicas puede diferenciar cuando las disminuciones de la amplitud o cambios de la latencia se deben a la maduración biológica y cuando a la eficiencia del proceso cognitivo.

Así, nos encontramos con varios avances pero también con problemáticas. Al respecto de los procesos de inhibición y de detección del conflicto nos encontramos con dos potenciales ampliamente estudiados, la N2 y la P3, queda aún por dilucidar la participación de cada uno de ellos en estos procesos cognitivos. Los resultados de los estudios revisados apuntan a que la disminución de la amplitud de la N2 se asocia a una mejora del proceso cognitivo durante el desarrollo, mientras que la P3 estaría más asociada a los procesos de inhibición de respuestas motoras.

En un paradigma tipo switch asociado al cambio atencional, al comparar la realización de tareas aisladas con tareas combinadas, se reporta una onda positiva que va aumentando su amplitud conforme el paso de la edad y que alcanza a las derivaciones frontales. Los autores sugieren que ésta se asocia a procesos de memoria de trabajo, mientras que la onda positiva centroparietal, no presenta cambios con el avance de la edad y estaría más relacionada a reconfiguración de la tarea.
Para el monitoreo del error se han estudiado los componentes ERN y $\mathrm{P}_{\mathrm{e}}$, el primero va apareciendo conforme se avanza en el desarrollo mientras que el segundo siempre está presente, está aún pendiente determinar la contribución de cada uno de ellos en el automonitoreo. Además, los PREs han aportado evidencia de que dicho proceso es más eficiente en niños que tienen mayor capacidad aeróbica.

También se revisó un estudio que demuestra que la escolaridad de la madre, como indicador del ESE, se asocia a menores ejecuciones en tareas de atención y a una amplitud similar de la onda positiva amplia entre los estímulos atendidos y los no atendidos lo que se interpretó como una ineficiencia para filtrar información irrelevante. Mientras que en la modalidad auditiva se encontró que los niños no presentaron una MMN sino una onda positiva y que la reorientación de la orientación reflejada por la onda RON se desarrolla hasta la edad adulta.

Considero que el estudio de las FE mediante el empleo de los PREs es un campo vasto cuyos hallazgos y aportaciones contribuirán a la explicación integral de los procesos cognitivos. Sin embargo, al trabajar en este campo se requiere de la declaración explícita de conceptualizaciones sobre lo que son o no son las FE, así como también del desarrollo de tareas creativas para la implementación de estudios robustos y prometedores. Quedando aún pendiente la exploración del proceso de establecimiento de metas y de la planeación como FE centrales.

\section{Agradecimientos}

Este trabajo se realizó con apoyo del proyecto CONACYT 25568 y del apoyo del PROMEP. 


\section{Artículos de Revisión}

\section{Referencias}

Anderson, V. (2001). Assessing executive functions in children: biological, psychological, and developmental considerations. Developmental Neurorehabilitation, 4(3), 119-136.

Baddeley, A. D., \& Hitch, G. J. (1974). Working memory. En G. H. Bower (Ed.). The psychology of learning and motivation, 8, 47- 89. New York: Academic Press.

Banfield, J. F., van der Lugt, A. H., \& Münte, T. F. (2006). Juicy fruit and creepy crawlies: an electrophysiological study of the implicit Go/NoGo association task. Neuroimage, 31(4), 1841-1849.

Barona, I. (2011). Estudio de la inhibición en niños y adolescentes mediante potenciales relacionados a eventos (Tesis). Cuernavaca, Mor. UAEM

Bentin, S., Allison, T., Puce, A., Perez, E., \& McCarthy, G. (1996). Electrophysiological studies of face perception in humans. Journal of Cognitive Neuroscience, 8, 551565.

Blair, C., Zelazo, P. D., \& Greenberg, M. T. (2005). The measurement of executive function in early childhood. Developmental neuropsychology, 28(2), 561-571.

Cabeza, R. (2002). Hemispheric asymmetry reduction in older adults: the HAROLD model. Psychology and aging, 17(1), 85.

Cepeda, N. J., Kramer, A. F. \& Gonzalez de Sather, J. C. (2001). Changes in executive control

Dawson, P. \& Guare, R. (2004). Executive skills in children and adolescents. New York: Guilford Press.

across the life span: examination of taskswitching performance. Developmental Psychology, 37(5), 715-730.

Ceponiene, R., Rinne, T., \& Näätänen, R. (2002). Maturation of cortical sound processing as indexed by event-related potentials. Clinical Neurophysiology, 113(6), 870-882.

Crone, E. A., Donohue, S. E., Honomichl, R., Wendelken, C., \& Bunge, S. A. (2006). Brain regions mediating flexible rule use during development. Journal of Neuroscience, 26 (43), 11239-11247.

Crone, E. A., Richard Ridderinkhof, K., Worm, M., Somsen, R. J., \& Van Der Molen, M. W. (2004). Switching between spatial stimulusresponse mappings: a developmental study of cognitive flexibility. Developmental science, 7(4), 443-455.

Davis, P. (1939) Effects of acoustic stimuli on the waking human brain. Journal of Neurophysiology, 2, 494-499.

Davis, E. P., Bruce, J., Snyder, K., \& Nelson, C. A. (2003). The X-trials: Neural correlates of an inhibitory control task in children and adults. Journal of Cognitive Neuroscience, 15(3), 432-443.

Davies, P. L., Segalowitz, S. J., \& Gavin, W. J. (2004). Development of Error-Monitoring Event-Related Potentials in Adolescents. Annals of the New York Academy of Sciences, 1021(1), 324-328. 


\section{Artículos de Revisión}

Diamond, A. (2002). Normal development of prefrontal cortex from birth to young adulthood: cognitive functions, anatomy, and biochemistry. In: Stuss, DT; Knight, RT., editors. Principles of Frontal Lobe Function. New York: Oxford University Press, 466-503.

Diamond, A., \& Lee, K. (2011). Interventions shown to aid executive function development in children 4 to 12 years old. Science, 333 (6045), 959-964.

Emslie, H., Wilson, F. C., Burden, V., NimmoSmith, I. \& Wilson, B. (2003). Behavioural assessment of dysexecutive syndrome for children. Bury St Edmunds, UK: Thames Valley Test Company.

Etnier, J. L., Salazar, W., Landers, D. M., Petruzzello, S. J., Han, M., \& Nowell, P. (1997). The influence of physical fitness and exercise upon cognitive functioning: A meta-analysis. Journal of Sport \& Exercise Psychology, 19, 249 -277.

Farah, M., Shera, D., Savage. J., Betancourt, L., Giannetta, J., Brodsky, N., et al. (2006). Childhood poverty: Specific associations with neurocognitive development. Brain Research, 1110, 166-174.

Ferrier, D. (1878). The Goulstonian Lectures On The Localisation Of Cerebral Disease (Concluded). The British Medical Journal, 443-447.

Finger, S. (2001). Origins of neuroscience: a history of explorations into brain function. USA: Oxford University Press.
Flores, J. C., Ostrosky, F., \& Lozano, A. (2012). Batería neuropsicológica de las funciones ejecutivas y lóbulos frontales (BANFE). México: Manual Moderno.

Garavan, H., Ross, T. J., Murphy, K., Roche, R. A. P., \& Stein, E. A. (2002). Dissociable executive functions in the dynamic control of behavior: inhibition, error detection, and correction. Neuroimage, 17(4), 1820-1829.

Gioia, G. A., Isquith, P. K., Roth, R. M., Guy, S. C., Kenworthy, L. \& Espy K. A. (2000). Behavior Rating Inventory of Executive Function. Manual. USA: PAR.

Haan, M., Pascalis, O., \& Johnson, M. (2002). Specialization of neural mechanisms underlying face recognition in human infants. Cognitive Neuroscience, Journal of, 14(2), 199-20

Hillman, C. H., Buck, S. M., Themanson, J. R., Pontifex, M. B., \& Castelli, D. M. (2009). Aerobic fitness and cognitive development: Event-related brain potential and task performance indices of executive control in preadolescent children. Developmental psychology, 45(1), 114.

Jonkman, L. M., Lansbergen, M., \& Stauder, J. E. A. (2003). Developmental differences in behavioral and event-related brain responses associated with response preparation and inhibition in a go/nogo task. Psychophysiology, 40, 752-761.

Kieffaber, P. D., \& Hetrick, W. P. (2005). Eventrelated potential correlates of taskswitching and switch costs. Psychophysiology, 42(1), 56-71. 
Korkman, M., Kirk, U., \& Kemp, S. (1998). NEPSY: A developmental neuropsychological assessment. San Antonio, TX; Psychological Corporation.

Kushnerenko, E., Ceponiene, R., Balan, P., Fellman, V., Huotilainen, M., \& Näätänen, R. (2002). Maturation of the auditory event-related potentials during the first year of life. Neuroreport, 13(1), 47-51.

Lamm, C., Zelazo, P. D., \& Lewis, M. D. (2006). Neural correlates of cognitive control in childhood and adolescence: Disentangling the contributions of age and executive function. Neuropsychologia, 44(11), 21392148 .

Lezak, M. (1982). The problem of assessing executive functions. International Journal of Psychology, 17, 281-297.

Luna, B., Thulborn, K. R., Munoz, D. P., Merriam, E. P., Garver, K. E., Minshew, N. J., ... \& Sweeney, J. A. (2001). Maturation of widely distributed brain function subserves cognitive development. Neuroimage, 13(5), 786-793.

Luria, A. R. (1980). Disturbances of higher cortical functions with lesions of the frontal region. In Higher cortical functions in man (246-365). US: Springer.

Luria, A. R. (1988). El cerebro en acción. Barcelona: Martínez Roca.

Matute, E., Roselli, M. \& Ardila, A. (2010). Evaluación Neuropsicológica Infantil. México: Manual Moderno.
Manzi, A., Nessler, D., Czernochowski, D., \& Friedman, D. (2011). The development of anticipatory cognitive control processes in task-switching: An ERP study in children, adolescents, and young adults. Psychophysiology, 48(9), 1258-1275.

Norman, D. A., \& Shallice, T. (1986) Attention to action: willed and automatic control of behavior. En R. J. Davidson, G. E. Schwartz y D. Shapiro, Consciousness and self-regulation, 4, 1-18. NY: Springer.

Nieuwenhuis, S., Yeung, N., Van den Wildenberg, W., \& Ridderinkhof, K. R. (2003). Electrophysiological correlates of anterior cingulate function in a $\mathrm{Go} / \mathrm{NoGo}$ task: Effects of response conflict and trialtype frequency. Cognitive, Affective \& Behavioral Neuroscience, 3, 17-26

O'Connell, R. G., Dockree, P. M., Bellgrove, M. A., Kelly, S. P., Hester, R., Garavan, H., ... \& Foxe, J. J. (2007). The role of cingulate cortex in the detection of errors with and without awareness: a high-density electrical mapping study. European Journal of Neuroscience, 25(8), 25712579 .

Picton, T. W. (1988). Human Eventrelated potentials. Handbook of electroencephalography and clinical neurophysiology. Amsterdam: Elsevier.

Polich, J. (1993). Cognitive brain potentials. Current Directions in Psychological Science, 175-179.

Polich, J., \& Kok, A. (1995). Cognitive and biological determinants of P300: An 


\section{Artículos de Revisión}

integrative review. Biological Psychology, 41,103-146.

Portellano, J. A., Martínez-Arias, R. \&Zumárraga, L. (2012). Evaluación neuropsicológica de las funciones ejecutivas en niños. Madrid: TEA ediciones.

Reimers, S.,\& Maylor, E. A. (2005). Task switching across the life span: effects of age on general and specific switch costs. Developmental psychology, 41(4), 661.

Ruchkin, D. S., Johnson Jr, R., Grafman, J., Canoune, H. \& Ritter, W. (1992). Distinctions and similarities among working memory processes: An eventrelated potential study. Cognitive Brain Research, 1(1), 53-66.

Ruhnau, P., Wetzel, N., Widmann, A. \& Schröger, E. (2010). The modulation of auditory novelty processing by working memory load in school age children and adults: a combined behavioral and event-related potential study. BMC neuroscience, 11(1), 126.

Sanders L, Stevens C, Coch, D. y Neville, H. (2006). Selective auditory attention in 3to 5-year-old children: An event-related potential study. Neuropsychologia, 44, 2126-2138.

Stevens, C., Sanders, L. \& Neville, H. (2006). Neurophysiological evidence for selective auditory attention deficits in children with specific language impairment. Brain Research, 1111, 143-152.

Stevens, C., Lauinger, B., \& Neville, H. (2009). Differences in the neural mechanisms of selective attention in children from different socioeconomic backgrounds: an event-related brain potential study. Developmental science, 12(4), 634-646.

Stuss, D. T., \& Benson, D. F. (1986). The frontal lobes. New York: Raven.

Sutton, S., Braren, M., Zubin, J., \& John, E. R. (1965). Evoked-potential correlates of stimulus uncertainty. Science, 150 (3700), 1187-1188. DOI:10.1126/ science.150.3700.1187.

Taylor, M. J., Batty, M., \& Itier, R. J. (2004). The faces of development: a review of early face processing over childhood. Journal of cognitive neuroscience, 16(8), 1426-1442.

Téllez-Alanís, B. (2013) David Ferrier y su contribución al entendimiento de la función prefrontal. Inventio, 17: 33-36.

Teuber, H. L. (2009). The riddle of frontal lobe function in man. Neuropsychology review, 19(1), 25-46.

Tieges, Z., Snel, J., Kok, A., Plat, N. \& Ridderinkhof, R. (2007). Effects of caffeine on anticipatory control processes: evidence from a cued task-switch paradigm. Psychophysiology, 44(4), 561-578.

Tranel, D., Anderson, S. W., \& Benton, A. (1994). Development of the concept of "executive function" and its relationship to the frontal lobes. En E. Boller y J. Grafman (Eds) Handbook of neuropsychology, 9, 125-148, Amsterdam: Elsevier Science. 


\section{Artículos de Revisión}

Walter, W. G., Cooper, R., Aldridge, V. J., McCallum, W. C., \& Winter, A. L. (1964). Contingent Negative Variation: An Electric Sign of Sensori-Motor Association and Expectancy in the Human Brain. Nature, 203(4943), 380384. doi:10.1038/203380a0

Welsh, M. C., Pennington, B. F., \& Groisser, D. B. (1991). A normative-developmental study of executive function: A window on prefrontal function in children. Developmental Neuropsychology, 7(2), 131-149.

Zelazo, P. D., \& Muller, U. (2002). Executive function in typical and atypical development. En: U. Goswami, editor. The Handbook of Cognitive Developmental Psychology. Oxford, UK: Blackwell. pp. 445-469. 